\title{
Extended field of view ultrasound imaging to evaluate Achilles tendon length and thickness: a reliability and validity study
}

\author{
Karin Gravare Silbernagel ${ }^{1}$ \\ Kristen Shelley ${ }^{2}$ \\ Stephen Powell2 \\ Shaun Varrecchia ${ }^{2}$ \\ 1 Department of Physical Therapy, University of \\ Delaware, Newark, USA \\ 2 Department of Physical Therapy for University of \\ the Sciences, Philadelphia, USA
}

Corresponding author:

Karin Gravare Silbernagel

Department of Physical Therapy, University of

Delaware

540 South College Avenue

19713 Newark, USA

E-mail: kgs@udel.edu

\section{Summary}

Background: Achilles tendon structural changes are common after injury and correlate with recovery of function. Having simple, inexpensive, yet valid and reliable measures of Achilles tendon structure are useful both in research and clinical. The purpose of this study was to perform reliability and validity measures of extended field of view (EFOV) ultrasound (US) imaging of the Achilles tendon.

Methods: eight cadavers (16 tendons) were used for the validation study to compare Achilles tendon length measurements from US images with actual measured length from dissected tendons. Nine healthy subjects (18 tendons) were included in the test-retest evaluation.

Results: the correlation between the US images and cadaveric measurements was excellent (ICC $=0.895)$ for the length between calcaneus and the gastrocnemius and good $(I C C=0.744)$ for the length between the calcaneus and the soleus. The between-limb reliability was excellent (ICC 0.886 0.940 ) for the tendon length measurements with standard error of measurements (SEM) of $0.64 \mathrm{~cm}$ for calcaneus to soleus and $0.67 \mathrm{~cm}$ for calcaneus to gastrocnemius. Between-day test-retest reliability was also excellent $(I C C=0.898-0.944)$.

Conclusion: this study supports the use of EFOV US imaging as a reliable and valid method to determine Achilles tendon length and thickness, and using the uninjured limb for comparison.
KEY WORDS: Achilles tendon rupture, triceps surae, tendon elongation, ultrasound imaging, extended field of view imaging.

\section{Introduction}

After an acute Achilles tendon rupture, there are large variations in outcomes between patients. Some patients have full recovery of function and no symptoms one year after injury, while others continue to have significant deficits in calf muscle strength and endurance on the injured side compared to the healthy side two years after injury ${ }^{1-4}$. The long-term deficits observed seem to not only be due to muscular deficits and insufficient rehabilitation, but also due to tendon elongation ${ }^{5-7}$. The occurrence of tendon elongation has also been reported to cause gait abnormalities both during walking and running, and correlates with patient reported symptoms $5,6,8$.

Various methods exist for measuring tendon length such as using radiographs and measuring the distance between metallic beads inserted into the tendon ${ }^{9}$, combining the use of ultrasound (US) imaging and motion analysis system ${ }^{10}$ or preforming a two step measure combining US imaging and tape measure ${ }^{11}$. The drawbacks with these methods are that they are either invasive, or require expensive and large equipment, or time consuming which is not very useful in clinical practice. There is also US imaging equipment that has the ability to perform extended field-of-view (EFOV) images by free-hand real-time scanning. These images allow for measurements of structures considerably longer then the length of the US transducer without having to combine with motion capture system. Previous studies have found EFOV US imaging to be accurate and reliable using acoustic phantoms ${ }^{12,13}$. Two other study found the EFOV US imaging to be reliable for evaluating the Achilles tendon length between the calcaneus and the myotendinous junction (MTJ) of the gastrocnemius ${ }^{14,15}$. However, we found no studies that have evaluated the validity of EFOV US imaging by comparing to a gold standard such as dissected tendon. Furthermore, we have not found any study evaluating the reliability of using the contralateral limb as a normative reference, or reliability for measuring the Achilles tendon length between the calcaneus and the soleus or the thickness using EFOV US imaging.

The purpose of this study was therefore to perform reliability and validity measures of EFOV US imaging of the Achilles tendon both by comparing the measure- 
ments from the images to actual tendon length of dissected Achilles tendons, and to perform inter-tester, between-limb and between-day test-retest reliability, and determine minimal detectable change of tendon length and thickness measurements.

\section{Materials and methods}

This study consisted of two parts. Eight cadavers (16 Achilles tendons) were used for validity measurements. Nine healthy subjects (18 Achilles tendons), mean (SD) age 22 (1.4) years, height $163(10) \mathrm{cm}$ and weight 70 (12) $\mathrm{kg}$, were evaluated twice two weeks apart for testretest reliability and stability measurements. A code was assigned to each subjects and cadaver, and used to identify the data and images. Each individual Achilles tendon was considered a separate subject. This study was approved by the Institutional Review Board at the University of the Sciences, Philadelphia, USA and meets the ethical standards of the Muscles, Ligaments and Tendons Journal ${ }^{16}$.

Healthy subjects were recruited through advertising at the University where the study was performed. All subjects were informed verbally and given written information regarding the purpose and procedure of this study prior to giving consent to participate. For the validation part of the study the measurements were done on 8 cadavers. The cadavers were used for a gross anatomy class. The cadavers were obtained through the Humanity Gifts Registry, the nonprofit organization that governs all anatomical donations in the Commonwealth of Pennsylvania. All cadavers were donated with the explicit purpose of medical education and research, and prior consent to this effect was given by the donor and/or next of kin.

\section{Ultrasound imaging}

All the US images were obtained using the LOGIQ e US (GE Healthcare) system using a wide-band linear array probe $(5.0-13.0 \mathrm{MHz})$. US images were recorded using B-mode at $10 \mathrm{mHz}$. The EFOV feature was used to obtain one picture that included both the calcaneus and the musculotendinous junction of the soleus and gastrocnemius as previously described by Ryan et al. ${ }^{15}$. Three images of each Achilles tendon were taken and saved on each occasion. All the length and thickness measurements were performed using the measurement tool on the US machine. Achilles tendon length was defined as the distance between the calcaneal osteotendinous junction and the musculo tendinous junction of soleus and gastrocnemius, aspreviously described in the literature ${ }^{15}$. The length of the tendon was measured in both the healthy subjects (Figs. 1, 2) and the cadavers. The tendon thickness was measured $2 \mathrm{~cm}$ proximal to the insertion on the calcaneus in the healthy individuals only (Fig. 3 ). The average of the

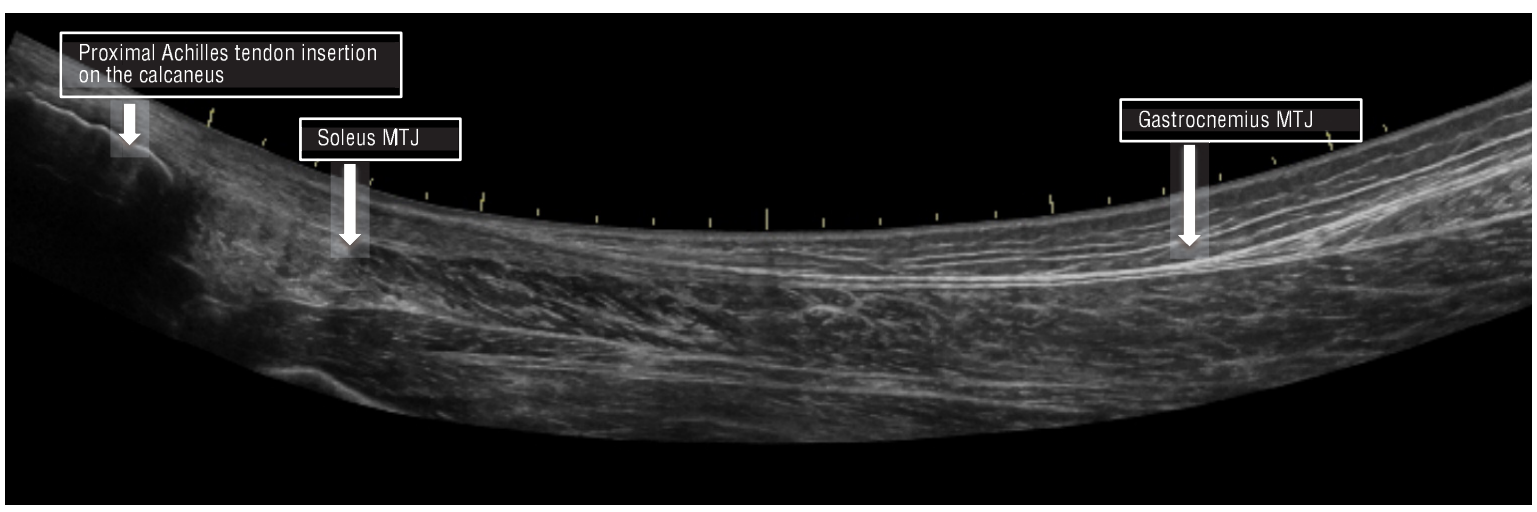

Figure 1. Start and end points for measuring Achilles tendon length on the ultrasound image.

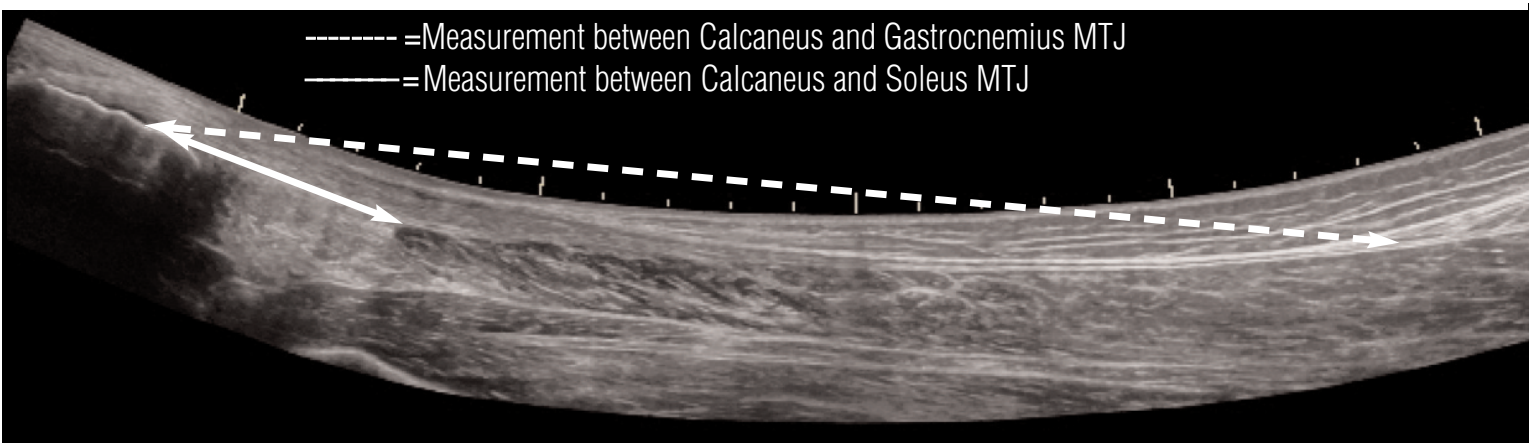

Figure 2. Measurements of Achilles tendon length between the calcaneus and Soleus and Gastrocnemius musculotendinous junctions (MTJ) on the ultrasound image. 


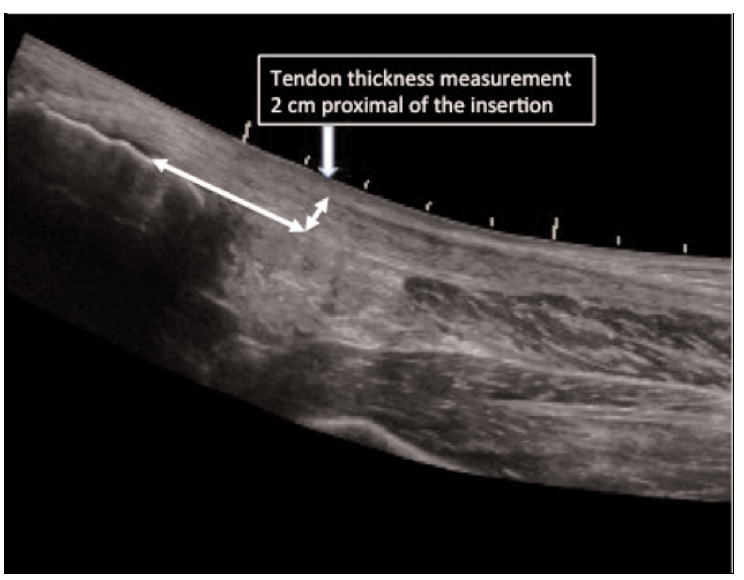

Figure 3. Measurement of Achilles tendon thickness on ultrasound image.

three measurements was used for calculation. One tester performed all the imaging on both the healthy individuals and the cadavers. The tester was a clinician who had 3 years of experience with ultrasound imaging. The healthy subjects were imaged in prone lying with the hip and knee straight and the ankle hanging off the treatment table. A slight tension was placed on the Achilles tendon by having the examiner stabilize the foot with one hand while taking the images with the other hand. The tester slowly moved the transducer from the heel in a straight line along the tendon and midportion of the calf. US images were taken on two testing days, two weeks apart for evaluating testretest reliability and stability of the measurements. The cadavers were measured when prone lying with the hip and knee in fixed extended position. The ankle angles varied in the cadavers. The US probe was covered in a disposable plastic sleeve.

\section{Anthropometric measurements of the cadavers' Achilles tendons}

The entire lower limbs of the cadavers were dissect- ed by the anatomy class. With the Achilles tendon exposed, tendon length from the calcaneal insertion to the musculotendinous junction of the soleus and the gastrocnemius (midline) was measured with a tape measure placed along the tendon.

\section{Statistical analysis}

All data were analyzed using the Statistical Package for Social Science (IBM Corp. Released 2011. IBM SPSS Statistics for Macintosh, Version 20.0. Armonk, NY: IBM Corp.) and Microsoft Excel. Descriptive data is reported as mean and standard deviation (SD). Inter-tester reliability of the measurement on the US images was performed by having two persons measure all the cadaver images independent of each other. Inter-tester reliability for measuring tendon length on the images, and between days test-retest reliability was analyzed with the intraclass correlation coefficient $\left(\mathrm{ICC}_{2,3}\right)$. ICC was also used to compare the measurements from the US images and the anthropometric measurements. Since the Achilles tendon length measurements are often used to compare an injured tendon with the same subject's healthy tendon a reliability analysis $\left(\mathrm{ICC}_{2,3}\right)$ comparing limbs was also performed using the data from test occasion one and two for the healthy subjects. According to the recommendations of Fleiss ${ }^{17}$, ICC values above 0.75 represent excellent reliability, values between 0.4 and 0.75 represent fair to good reliability, while values below 0.4 represent poor reliability. A repeated measures ANOVA with Greenhouse-Geisser correction was used to compare the measurements on the US images and the anthropometric measurements. A paired t-test was used to evaluate if there were any differences between the measurements on day 1 and day 2 , or between the right and left side in the healthy individuals. Standard error of measurements (SEM) was calculated as follows: SEM $=S D \sqrt{ }$, where SD is the standard deviation of the baseline measurements ${ }^{18}$. The minimal detectable change ( $\left.\mathrm{MDC}_{95 \%}\right)$

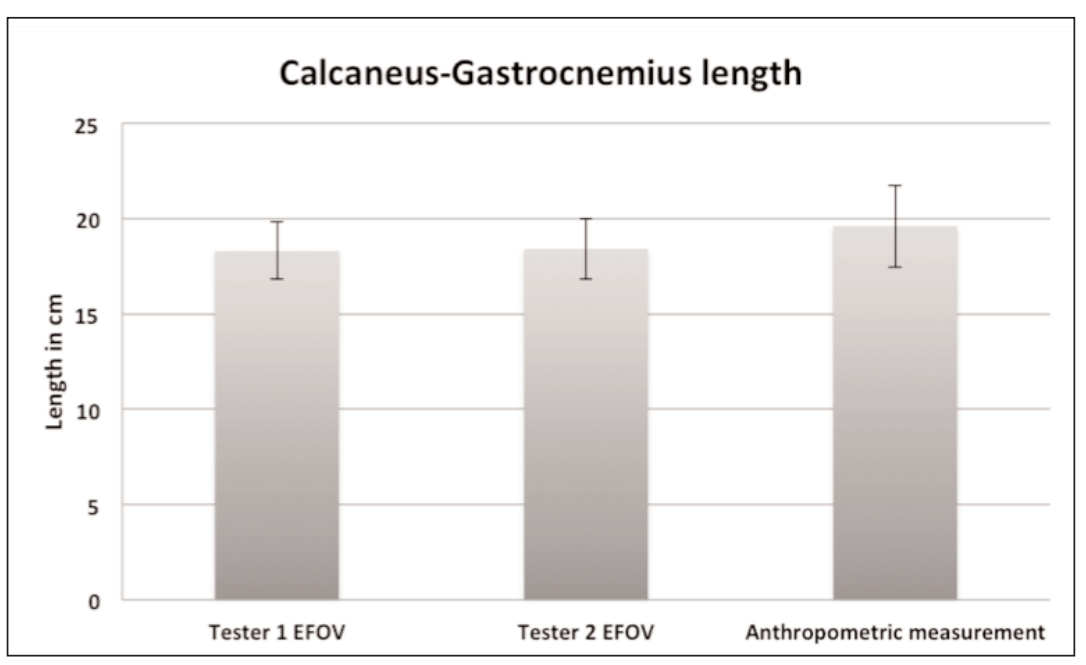

Figure 4. Mean (SD) tendon length from Achilles tendon insertion to calcaneus to gastrocnemius MTJ measured by EFOV US imaging and physical measurement of cadaver specimen. Two different testers measured the US images (The error bars indicate the SD). 


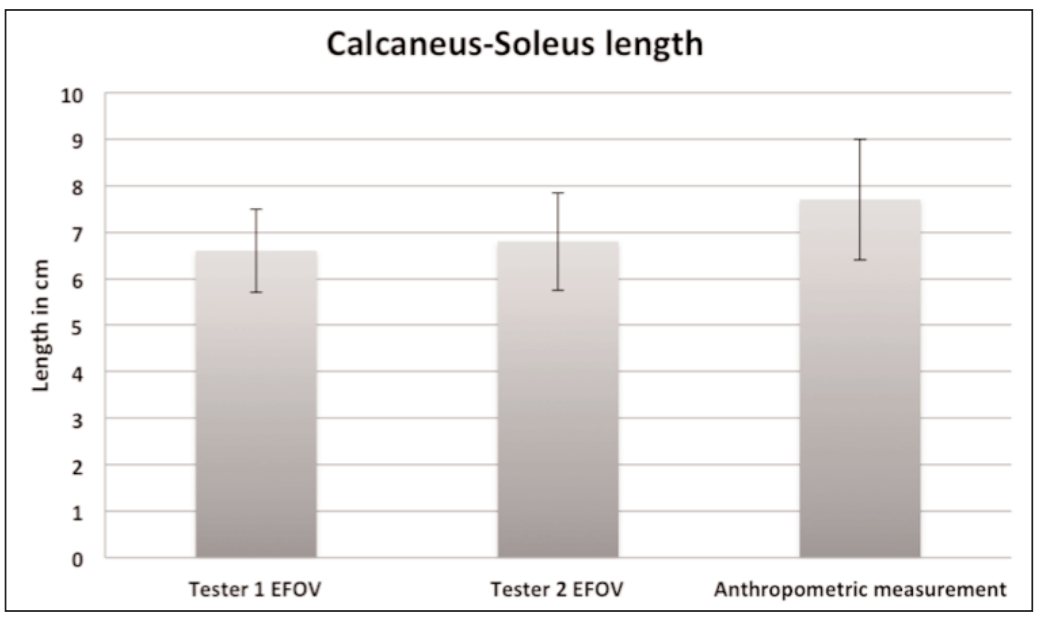

Figure 5. Mean (SD) tendon length from Achilles tendon insertion to calcaneus to soleus MTJ measured by EFOV US imaging and physical measurement of cadaver specimen. Two different testers measured the US images (The error bars indicate the SD).

was calculated as follows: for individual level $\mathrm{MDC}_{95 \%}=2.77 \times$ SEM and group level MDC $_{95 \%}=2.77$ $\mathrm{x} S E M / \sqrt{ } \mathrm{n}$. The level of significance was set at $\mathrm{p}<0.05$.

\section{Results}

Comparison between EFOV US imaging and anthropometric measurements

There were no significant differences $(p=0.15)$ between the length measurements on the US images (two testers) and the anthropometric measurements on the cadaver (Figs. 4, 5). The relationship between the EFOV US images and physical measurements was excellent $(I C C=0.895)$ for the length between calcaneus and the MTJ of the gastrocnemius and good $(I C C=0.744)$ for the length between the calcaneus and the MTJ of the soleus.

\section{Inter-rater reliability for measurement on the US images}

Inter-rater reliability for measuring tendon lengths on the US images was excellent (ICC 0.987-0.997) (Tab. 1).

\section{Between limb reliability}

There was no significant difference in the Achilles
Table 2. The between limb reliability and standard error of measurement (SEM).

\begin{tabular}{lll}
\hline & SEM cm & ICC (95\%Cl) \\
\hline Calcaneus to soleus & 0.64 & $\begin{array}{l}0.886 \\
(0.694-0.957)\end{array}$ \\
Calcaneus to gastrocnemius & 0.67 & $\begin{array}{l}0.940 \\
(0.840-0.978)\end{array}$ \\
Achilles tendon thickness & 0.02 & $\begin{array}{l}0.781 \\
\end{array}$ \\
& & $(0.414-0.918)$
\end{tabular}

$\mathrm{Cl}=$ confidence interval, $\mathrm{ICC}=$ intraclass correlation coefficient, SEM=standard error of measurement.

tendon length measurements between limbs and the measurements had excellent reliability (ICC 0.8860.940) (Tab. 2). For the Achilles tendon thickness measurement there was a significant $(p=0.043)$ difference between limbs however the measurement had excellent reliability (ICC 0.781) (Tab. 2).

\section{Between days test-retest reliability}

There were no significant differences in any of the length or thickness measurements between day 1 and day 2 , and the measurements had excellent (ICC $0.898-0.944$ ) reliability in the healthy individuals (Tab. $3)$. The minimal detectable change at the individual and group level is presented in Table 3.

Table 1. Inter-rater reliability for measuring tendon length on EFOV US images.

\begin{tabular}{llll}
\hline & Tester $\mathbf{1}$ & Tester $\mathbf{2}$ & ICC \\
\hline Calcaneus to soleus & 6.59 & 6.77 & 0.987 \\
Mean $(S D)$ cm & $(+1.85)$ & $(+2.11)$ & \\
Calcaneus to gastrocnemius & 18.3 & 18.44 & 0.997 \\
Mean $(S D)$ cm & $(+3.05)$ & $(+3.17)$ & \\
\hline
\end{tabular}

$\mathrm{ICC}=$ intraclass correlation coefficient, $\mathrm{SD}=$ standard deviation. 
Table 3. Test-retest reliability for imaging and measuring tendon length on two separate occasions in 18 healthy Achilles tendons using EFOV US imaging.

\begin{tabular}{llllllll}
\hline & $\begin{array}{l}\text { Day 1 } \\
\text { Mean (SD) } \\
\mathbf{c m}\end{array}$ & $\begin{array}{l}\text { Day 2 } \\
\text { Mean (SD) } \\
\mathbf{c m}\end{array}$ & $\begin{array}{l}\text { Diff. Mean } \\
\mathbf{( c m )}\end{array}$ & $\begin{array}{l}\text { SEM } \\
\mathbf{( c m )}\end{array}$ & $\begin{array}{l}\text { ICC } \\
\mathbf{( 9 5 \% C l )}\end{array}$ & $\begin{array}{l}\text { MDC }_{95 \%} \\
\text { individual }\end{array}$ & $\begin{array}{l}\text { MDC } \\
\text { group }\end{array}$ \\
\hline $\begin{array}{l}\text { Calcaneus to soleus } \\
\text { gm }\end{array}$ & $6.02(1.96)$ & $5.76(1.61)$ & 0.25 & 0.6 & $\begin{array}{l}0.898 \\
(0.728-0.962)\end{array}$ & 1.73 & 0.41 \\
$\begin{array}{l}\text { Calcaneus to } \\
\text { gastrocnemius }\end{array}$ & $19.63(2.78)$ & $19.13(2.78)$ & 0.51 & 0.7 & $\begin{array}{l}0.944 \\
(0.852-0.979)\end{array}$ & 1.83 & 0.43 \\
$\begin{array}{l}\text { Achilles tendon } \\
\text { thickness }\end{array}$ & $0.429(0.034)$ & $0.433(0.046)$ & 0.004 & 0.01 & $\begin{array}{l}0.898 \\
(0.728-0.962)\end{array}$ & 0.03 & 0.007 \\
\hline
\end{tabular}

$\mathrm{Cl}=$ confidence interval, ICC=intraclass correlation coefficient, $\mathrm{MDC}=$ minimal detectable change, $\mathrm{SD}=$ standard deviation, SEM=standard error of measurement.

\section{Discussion}

The main purpose of this study was to evaluate the reliability and validity of using EFOV US imaging to measure Achilles tendon length and thickness. The Achilles tendon length measurements performed on the images using the US system tool were not significantly different and had good to excellent correlation to the anthropometric measurements of the dissected Achilles tendons. Furthermore the inter-rater reliability of performing the measurements on the images was excellent. The between limb and between day test-retest reliability in healthy individuals was also excellent. The SEM for comparing limbs are also considerably less than the reported differences between healthy and injured sides in patients with Achilles tendon rupture ${ }^{10}$. This study therefore supports the use of EFOV US imaging as a reliable, valid and simple method to measure Achilles tendon structure.

\section{Validity}

An unique aspect of this study is the use of dissected tendons as a gold standard for determining the validity of the Achilles tendon length measurements. A similar method was previously performed for patellar tendon measurements with the use of 4 cadaveric knees ${ }^{19}$. Our study was able to include a larger sample size with a total of 16 cadaveric tendons. One of the difficulties with the EFOV US imaging of the cadavers' tendons was the rigidity of the ankle, which varied in position from $20-65^{\circ}$ of plantar flexion. With a greater degree of plantar flexion there was a greater the curvature of the tendon seen on the EFOV US images. Since all the measurements on the US images were performed with the system's straight-line measurement tool the Achilles tendon lengths might have been underestimated in the cadavers (Fig. 6). Our results indicate that the anthropometric measurements were larger, but there were no significant differences between the images and anthropometric measurements in this sample. Also the length measurements to the soleus MTJ did not show as strong correlation as the measurement to the gastrocnemius MTJ when comparing the images with the cadavers. This was probably partially due to difficulty determining the exact location of the soleus MTJ in the cadavers. It was easier to visualize the gastrocnemius MTJ during dissection and we felt more confident in those measurements.

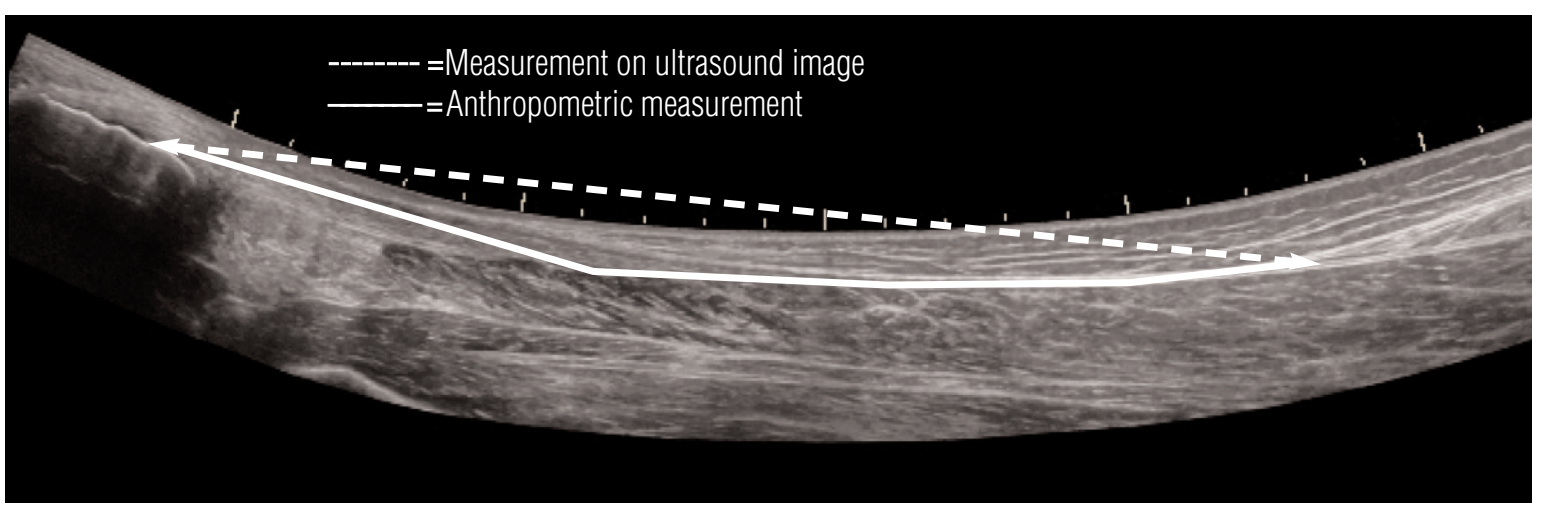

Figure 6. Comparison of Achilles tendon length between measurement on ultrasound image and the anthropometric measurement. 
Despite these difficulties the EFOV US image measurements had excellent correlation with the cadavers indicating that this is a valid method for evaluating Achilles tendon length.

\section{Inter-rater reliability}

Performing US imaging requires that the user is trained in the technique and therefore it is often the same person taking the images and measuring them, whether in research studies or in the clinic. To minimize the bias of the method it is beneficial to have another person measure the US images. In this study we compared the inter-rater reliability between two persons who performed the measurements on the US images independent of one another. Both persons were trained to read US images of the Achilles tendon in one session that focused on how to find the Achilles tendon insertion on the calcaneus and the MTJs of the gastrocnemius and soleus. The length measurements had excellent inter-rater reliability, which indicates that different individuals can extract the length data from US images.

\section{Between limb reliability}

Patients with Achilles tendon rupture have been found to have elongation of the injured tendon compared to their healthy side $e^{6,10}$. The degree of tendon elongation also has been found to correlate with functional changes $^{10}$ and patient reported outcomes ${ }^{6}$. Healthy individuals do not have a side-to-side difference in tendon length, however there are large variations in tendon lengths between individuals ${ }^{10}$. To perform comparisons between the patient's healthy and injured side is therefore at this time the best way to determine the degree of elongation after injury. The results from this study found that the SEM of the Achilles tendon length measurements $(0.64$ and 0.67 $\mathrm{cm}$ ) are considerably less than the difference found between the injured and healthy side $(2.6-3.1 \mathrm{~cm})$ in patients with an Achilles tendon rupture ${ }^{10}$. This indicates that these measurements can be used to compare limbs and determine the degree of tendon elongation after injury.

\section{Between-day reliability}

US imaging may be used clinically and in research to evaluate how the Achilles tendon elongates after an Achilles tendon rupture. This requires repeated US scans over time. To determine the full utility of this method, it is important to understand if the tendon length changes over time in healthy individuals, as well as to determine the test-retest reliability of the method. In this study, the same individual imaged the healthy subjects twice, two weeks apart. We did not control for the subjects' activity levels norfor the time of day images were taken. There were no significant differences in either of the length measurements nor in the tendon thickness measurements between the two testing dates indicating that this is a stable metric in healthy subjects. The test-retest reliability was excellent and similar to that of other studies that have measured Achilles tendon length between calcaneus and gastrocnemius MTJ10,11,14,15. The reported MDC values are also informative concerning what value for change over time can be considered as a true difference. The results from this study supports that these simple measurements are both reliable and can be used to measure Achilles tendon structure over time.

\section{Limitations}

The main limitation in this study is that the test-retest measurements were performed in healthy individuals. The reason for this was that we were interested in studying if these measurements were stable or if they changed over time in healthy individuals. The length measurement to the gastrocnemius MTJ has previously been performed in patients with Achilles tendon rupture by Silbernagel et al. ${ }^{10}$ and, as in the current study, there was no difficulty in determining the Achilles tendon insertion on the calcaneus or the MTJ of the gastrocnemius. On some of the patients with an Achilles tendon rupture it is however, very difficult to determine the soleus MTJ and we recommend that further reliability studies be performed for this measurement in injured subjects. Another limitation is that we used the US system's straight-line measuring tool, which does not accommodate for the curvature on the image. This then might underestimate the actual length of the tendon and if the absolute length is of importance we recommend exporting the images and measure in another software such as Image $\mathrm{J}^{20}$.

\section{Clinical implication}

Achilles tendon rupture is a devastating injury that causes long lasting difficulties being physically active and returning to sport ${ }^{21-23}$. One third of professional football players with an Achilles tendon rupture never return to the professional level and those who return have a $50 \%$ reduction in performance ${ }^{24}$. Similar results are found in professional basketball players ${ }^{25}$. One of the main reason for remaining deficits in function seems to be that the tendon heals in an elongated position ${ }^{6-8}$. This elongation causes a decrease in the plantar flexion force because the muscle is acting at a different position along the force-length curve ${ }^{8}$. To understand how to minimize the elongation and evaluate what intervention addresses this best we need to have methods that can evaluate tendon length and thickness accurately. In randomized clinical trials on treatment after an Achilles tendon rupture the normal sample size is around 100 subjects $^{1,2}$. It is therefore of value to have a method that is simple, inexpensive and fast that can measure tendon lengths in large samples. Furthermore, Achilles tendon thickness has been used to evaluate tendon health ${ }^{26}$ and increased thickness has been found to be a risk factor for Achilles tendinopathy ${ }^{27}$. Using EFOV US images seems to be very well suited for evaluating tendon structure and this study supports its validity and reliability. 


\section{Conflict of interests}

The Authors declare that they have no conflict of interests regarding the publication of this paper.

\section{References}

1. Nilsson-Helander K, Silbernagel KG, Thomeé R, et al. Acute achilles tendon rupture: a randomized, controlled study comparing surgical and nonsurgical treatments using validated outcome measures. Am J Sports Med. 2010;38(11):2186-2193.

2. Olsson N, Silbernagel KG, Eriksson BI, et al. Stable surgical repair with accelerated rehabilitation versus nonsurgical treatment for acute Achilles tendon ruptures: a randomized controlled study. Am J Sports Med. 2013;41(12):2867-2876.

3. Willits K, Amendola A, Bryant D, et al. Operative versus nonoperative treatment of acute Achilles tendon ruptures: a multicenter randomized trial using accelerated functional rehabilitation. J Bone Jt Surg Am. 2010;92(17):2767-2775.

4. Khan RJ, Carey Smith RL. Surgical interventions for treating acute Achilles tendon ruptures. Cochrane Database Syst Rev. 2010;(9):CD003674.

5. Don R, Ranavolo A, Cacchio A, et al. Relationship between recovery of calf-muscle biomechanical properties and gait pattern following surgery for achilles tendon rupture. Clin Biomech (Bristol, Avon). 2007;22(2):211-220.

6. Kangas J, Pajala A, Ohtonen P, Leppilahti J. Achilles tendon elongation after rupture repair: a randomized comparison of 2 postoperative regimens. Am J Sport Med. 2007;35(1):59-64.

7. Mullaney MJ, McHugh MP, Tyler TF, Nicholas SJ, Lee SJ. Weakness in end-range plantar flexion after Achilles tendon repair. Am J Sport Med. 2006;34(7):1120-1125.

8. Suydam SM, Buchanan TS, Manal K, Silbernagel KG. Compensatory muscle activation caused by tendon lengthening post-Achilles tendon rupture. Knee Surg Sports Traumatol Arthrosc. 2013:1-7.

9. Schepull T, Kvist J, Andersson C, Aspenberg P. Mechanical properties during healing of Achilles tendon ruptures to predict final outcome: a pilot Roentgen stereophotogrammetric analysis in 10 patients. BMC Musculoskelet Disord. 2007;8:116.

10. Silbernagel KG, Steele R, Manal K. Deficits in heel-rise height and achilles tendon elongation occur in patients recovering from an achilles tendon rupture. Am J Sport Med. 2012;40(7): 1564-1571.

11. Barfod KW, Riecke AF, Boesen A, et al. Validation of a novel ultrasound measurement of achilles tendon length. Knee Surg Sports Traumatol Arthrosc. 2015;23(11):3398-3406.

12. Ying $\mathrm{M}, \operatorname{Sin} \mathrm{MH}$. Comparison of extended field of view and dual image ultrasound techniques: Accuracy and reliability of distance measurements in phantom study. Ultrasound Med
Biol. 2005;31(1):79-83.

13. Weng L, Tirumalai AP, Lowery CM, et al. US extended-field-ofview imaging technology. Radiology. 1997;203(3):877-880.

14. Stokes OM, Theobald PS, Pugh ND, Nokes LDM. Panoramic ultrasound to measure in vivo tendo Achilles strain. Foot ankle Int. 2010;31(10):905-909.

15. Ryan ED, Rosenberg JG, Scharville MJ, Sobolewski EJ, Thompson BJ, King GE. Test-Retest Reliability and the Minimal Detectable Change for Achilles Tendon Length: A Panoramic Ultrasound Assessment. Ultrasound Med Biol. 2013;39 (12):2488-2491.

16. Padulo J, Oliva F, Frizziero A, Maffulli N. Muscles, Ligaments and Tendons Journal. Basic principles and recommendations in clinical and field science research. MLTJ. 2013;3(4):250-252.

17. Fleiss $\mathrm{JL}$. The Design and Analysis of Clinical Experiments. New York: Wiley; 1986.

18. Denegar CR, Ball DW. Assessing Reliability and Precision of Measurement: An introduction to Intraclass Correlation and Standard Error of Measurement. J Sport Rehab. 1993;2(1):3542.

19. Gellhorn AC, Morgenroth DC, Goldstein B. A Novel Sonographic Method of Measuring Patellar Tendon Length. Ultrasound Med Biol. 2012;38(5):719-726.

20. Abràmoff MD, Magalhães PJ, Ram SJ. Image processing with imageJ. Biophotonics Int. 2004;11(7):36-41.

21. Keating JF, Will EM. Operative versus non-operative treatment of acute rupture of tendo Achillis: a prospective randomised evaluation of functional outcome. J Bone Jt Surg Br. 2011;93(8):1071-1078.

22. Olsson N, Nilsson-Helander K, Karlsson J, et al. Major functional deficits persist 2 years after acute Achilles tendon rupture. Knee Surgery, Sport Traumatol Arthrosc. 2011;19(8): 1385-1393.

23. Cetti R, Christensen SE, Ejsted R, Jensen NM, Jorgensen U. Operative versus nonoperative treatment of Achilles tendon rupture. A prospective randomized study and review of the literature. Am J Sport Med. 1993;21(6):791-799.

24. Parekh SG, Wray 3rd WH, Brimmo O, Sennett BJ, Wapner $\mathrm{KL}$. Epidemiology and outcomes of Achilles tendon ruptures in the National Football League. Foot Ankle Spec. 2009;2(6): 283-286.

25. Amin NH, Old AB, Tabb LP, Garg R, Toossi N, Cerynik DL. Performance outcomes after repair of complete achilles tendon ruptures in national basketball association players. Am J Sports Med. 2013;41(8):1864-1868.

26. Abate M, Oliva F, Schiavone C, Salini V. Achilles tendinopathy in amateur runners: role of adiposity (Tendinopathies and obesity). Muscles Ligaments Tendons J. 2012;2(1):44-48.

27. Jhingan S, Perry M, O'Driscoll G, et al. Thicker Achilles tendons are a risk factor to develop Achilles tendinopathy in elite professional soccer players. Muscles Ligaments Tendons $\mathrm{J}$. 2011;1(2):51-56. 